\title{
On Imprecise Investment Recommendations
}

\section{Krzysztof Piasecki ${ }^{1}$}

1 Department of Operations Research, Poznań University of Economics, Poland, k.piasecki@ue.poznan.pl

\begin{abstract}
The return rate is considered here as a fuzzy probabilistic set. Then the expected return is obtained as a fuzzy subset in the real line. This result is a theoretical foundation for new investment strategies. All considered strategies result of comparison profit fuzzy index and limit value. In this way we obtain an imprecise investment recommendation. Financial equilibrium criteria are a special case of comparison of the profit index and the limit value. The following criteria are generalized here: the Sharpe's Ratio, the Jensen's Alpha and the Treynor's Ratio. Moreover, the safety-first criteria are generalized here for the fuzzy case. The Roy Criterion, the Kataoka Criterion and the Telser Criterion are also generalized. Obtained results show that proposed theory is useful for the investment applications.
\end{abstract}

\section{Research problem}

In the interest theory, the present value (PV) is defined as discounted cash flow. In general case, PV may be defined as the utility of cash flow (Piasecki, 2012). Behavioral reasons cause subjective nature of this utility (Dacey, Zielonka, 2005). Imprecise estimation of PV is a result of the subjective approach to security valuation (Barberis et al., 1998). This is the main reason for the description of present value as a fuzzy number.

The suggestion to present the financial statements as fuzzy sets derives from (Ward, 1985), (Calzi, 1990). Ward (1995) defines fuzzy PV as discounted fuzzy cash flow. The fuzzy cash flow used here is interpreted as an imprecise forecast of future crisp cash flow. The Ward's definition is generalized to the case of fuzzy duration in (Greenhut et al., 1995). Sheen (1995) generalizes Ward's definition to the case of fuzzy interest rate. Some problems connected with the application of fuzzy arithmetic for calculating fuzzy PV are discussed in (Buckley, 1987, 1992), (Gutierrez, 1989), (Kuchta, 2000) and (Lesage, 2001). Huang (2007) generalizes Ward's definition for the case when future cash flow is given as a fuzzy random variable (Kwakernaak, 1978, 1979). A more general definition of fuzzy PV is proposed by 
Tsao (2005) who assumes that future cash flow is a fuzzy probabilistic set (Hiroto, 1981). In (Piasecki, 2011a, b) fuzzy PV is defined as a fuzzy number dependent on current market price, equilibrium price and sentiment index (Edwards, 1968), (Barberis et al., 1998). Next, in (Piasecki, 2013) the last model was generalized to the case when PV is described by the fuzzy number dependent only on the market price.

In agreement with uncertainty thesis (Mises, 1962), (Kaplan et al., 1967), each future cash flow is under uncertainty. Then future value (FV) is usually defined as a random variable. Therefore, if PV is a fuzzy number then return rate is the fuzzy probabilistic set (Hiroto, 1981). For this case, expected return rate is given as fuzzy subset in the real line $\mathbb{R}$ (Piasecki, $2011 b, c)$. This means that the financial market states are described imprecisely. It implies that investment goals are formulated imprecisely (Fang Yong; et al., 2008). Each security with fuzzy probabilistic return is at imprecision risk and at uncertainty risk. The knightian uncertainty (Knight, 1921) is excluded of course.

The main goal of this article is consideration of investment recommendations given for securities with fuzzy probabilistic return. We will consider the financial equilibrium criteria and the safety-first criteria. In this way, when setting investment goal we will be able to take into account the imprecision risk.

\section{Fuzzy probabilistic return}

Let us assume that the time horizon $t>0$ of an investment is fixed. Then, any security is determined by two values:

- anticipated future value $V_{t} \in \mathbb{R}^{+}$,

- assessed present value $V_{0} \in \mathbb{R}^{+}$.

The basic characteristic of benefits from owning this instrument is a rate of return $r \in \mathbb{R}$ given by the relationship

$$
r=r\left(V_{0}, V_{t}\right) .
$$

In the general case, the function $r: \mathbb{R}^{+} \times \mathbb{R}_{0}^{+} \rightarrow \mathbb{R}$ is strictly decreasing function of present value. It implies that for any fixed future value $V_{t}$ we can determine inverse function $r_{0}^{-1}\left(\cdot, V_{t}\right): \mathbb{R} \rightarrow \mathbb{R}^{+}$.

The future value is at risk of uncertainty. A formal uncertainty model is the presentation of future value $V_{t}$ as a random variable $\tilde{V}_{t}: \Omega \rightarrow \mathbb{R}^{+}$, where $\Omega=\{\omega\}$ is a set of financial market states. In the classical approach to the problem of return rate determination, security PV is identified with 
the current market price $\check{C}$. Then the return rate is a random variable which is at uncertainty risk. This random variable is determined by the identity

$$
\tilde{r}(\omega)=r\left(\check{C}, \tilde{V}_{t}(\omega)\right) .
$$

In practice of financial markets analysis, the uncertainty risk is usually described by probability distribution of return rates. Let us assume that this probability distribution is given by cumulative distribution function $F: \mathbb{R} \rightarrow[0 ; 1]$. In the case of Warsaw Stock Exchange, the normal inverse Gaussian (NIG) distribution is the best matching probability distribution of logarithmic returns (Piasecki, Tomasik, 2013). For any random variable with NIG distribution its variance exists (Bølviken, Benth, 2000). On the other hand, any return rate is increasing function of logarithmic return (Piasecki, 2013). Thus we can assume that return variance $\sigma^{2}$ exists. Then, the uncertainty risk may be characterized by this variance.

The probability distribution of $\mathrm{FV}$ is described by conditional cumulative distribution function $F_{V}(\cdot \mid \check{C}): \mathbb{R}^{+} \rightarrow[0 ; 1]$ determined in the following way

$$
F_{V}(x \mid \check{C})=F(r(\check{C}, x)) .
$$

The PV of security is approximately equal to security market price $\check{C}$. Thus it may be at imprecision risk. Then the security PV is described by fuzzy number (Dubois, Prade 1979) dependent on the market price $\check{C}$. Each PV membership function $\mu(\cdot \mid \check{C}): \mathbb{R}^{+} \rightarrow[0 ; 1]$ fulfils the following properties

$$
\begin{gathered}
\mu(\check{C} \mid \check{C})=1 \\
\forall_{x, y, z \in \mathbb{R}^{+}}: x \leq y \leq z \Rightarrow \mu(y \mid \check{C}) \geq \min \{\mu(z \mid \check{C}), \mu(x \mid \check{C})\} .
\end{gathered}
$$

Each investor takes into account the lowest possible market price and the highest one. The security PV should be greater than the lowest possible price. Also, the security PV should be less than the highest possible price. Therefore, about any PV membership function $\mu(\cdot \mid \check{C}): \mathbb{R}^{+} \rightarrow[0 ; 1]$ we assume additionally that it fulfils the following condition

$$
\begin{gathered}
\forall{\check{C} \in \mathbb{R}^{+}}_{\check{C}_{\min }, \check{C}_{\max } \in \mathbb{R}^{+}}: \check{C}_{\min }<\check{C}<\check{C}_{\max } \wedge \\
\wedge \mu\left(\check{C}_{\min } \mid \check{C}\right)=\mu\left(\check{C}_{\max } \mid \check{C}\right)=0 .
\end{gathered}
$$

Immediately from (5) and (6) we obtain

$$
\forall_{\check{C} \in \mathbb{R}^{+}} \forall_{x \in \mathbb{R}^{+}}: x \notin\left(\check{C}_{\min }, \check{C}_{\max } \Rightarrow \wedge \mu(x \mid \check{C})=0\right.
$$


The return rate is then at risk of coincidence uncertainty and imprecision. According to the Zadeh extension principle, for each fixed elementary state $\omega \in \Omega$ of financial market, membership function $\rho(\cdot, \omega \mid \check{C}): \mathbb{R} \rightarrow[0 ; 1]$ of return rate is determined by the identity

$$
\begin{aligned}
\rho(r, \omega \mid \check{C}) & =\max \left\{\mu(y \mid \check{C}): y \in \mathbb{R}^{+}, r=r\left(y, \tilde{V}_{t}(\omega)\right)\right\}= \\
& =\mu\left(r_{0}^{-1}\left(r, \tilde{V}_{t}(\omega)\right) \mid \check{C}\right) .
\end{aligned}
$$

It means that the return rate considered here is represented by fuzzy probabilistic set (Hiroto, 1981). For this reason, this return rate is called fuzzy probabilistic return.

For any fuzzy probabilistic return we define the expected return distribution as follows

$$
\varrho(r \mid \check{C})=\int_{-\infty}^{+\infty} \mu\left(r_{0}^{-1}(r, x) \mid \check{C}\right) d F_{V}(x \mid \check{C}) .
$$

The function $r_{0}^{-1}(r, \cdot): \mathbb{R}_{0}^{+} \rightarrow \mathbb{R}^{+}$is increasing one. Therefore, the condition (7) implies that

$$
\begin{gathered}
\forall_{\check{C} \in \mathbb{R}^{+}} \exists_{x_{\min }, x_{\max } \in \mathbb{R}_{0}^{+}} \forall_{x \in \mathbb{R}_{0}^{+}}: x \notin\left(x_{\min }, x_{\max }\right) \Rightarrow \\
\Rightarrow \wedge \mu\left(r_{0}^{-1}(r, x) \mid \check{C}\right)=0
\end{gathered}
$$

It proves that expected return distribution always exists. Distribution of expected return $\varrho(\cdot \mid \check{C}): \mathbb{R} \rightarrow[0 ; 1]$ is a membership function of fuzzy subset $\tilde{R}$ in the real line. This subset is imprecise assessment of the expected return value. Therefore, it is called fuzzy expected return. In general case, fuzzy expected return is not any fuzzy number. In this way, any security with fuzzy probabilistic return is represented by pair $\left(\varrho(\cdot \mid \check{C}), \sigma^{2}\right)$.

\section{Investment recommendations dependent on expected return}

The investment recommendation is the counsel given by the advisers to the investor. For convenience, these recommendations may be expressed by means of standardized ratings. Here we will consider the following advisers vocabulary:

- Buy suggesting that evaluated security is significantly undervalued,

- Accumulate suggesting that evaluated security is undervalued,

- Hold suggesting that evaluated security is fairly valued,

- Reduce suggesting that evaluated security is overvalued,

- Sell suggesting that evaluated security is significantly overvalued. 
Many advisers use a different terminology and different number of words forming advice vocabulary. ${ }^{1}$ In this article we will concentrate on the five-element adviser's vocabulary. Recommendations mentioned above form the set

$$
\mathbb{A}=\left\{A^{++}, A^{+}, A^{0}, A^{-}, A^{--}\right\}
$$

called advisers' vocabulary, where

- $A^{++}$denotes the advice Buy,

- $A^{+}$denotes the advice Accumulate,

- $A^{0}$ denotes the advice Hold,

- $A^{-}$denotes the advice Reduce,

$-A^{--}$denotes the advice Sell.

Let us take into account fixed security $\breve{S}$ represented by the pair $\left(r_{s}, \sigma_{s}^{2}\right)$ where:

- $r_{s}$ denotes expected return on $\check{S}$,

- $\sigma_{s}^{2}$ denotes variance of return on $\check{S}$.

In such case adviser's counsel depends on expected return and return variance. Then the criterion for competent choice of advice can be presented as a comparison of the values $g\left(\bar{r} \mid \sigma_{s}^{2}\right)$ and $\hat{G}$ defined as follows:

- $g\left(\cdot \mid \sigma_{s}\right): \mathbb{R} \rightarrow \mathbb{R}$ is an increasing function in substantially justified form,

- $\hat{G}$ denotes the substantially justified limit value.

The function $g\left(\cdot \mid \sigma_{s}\right): \mathbb{R} \rightarrow \mathbb{R}$ serves as a profit index. Using this criterion we define the advice choice function $\Lambda: \mathbb{R}^{2} \rightarrow 2^{\mathbb{A}}$ as follows

$$
\left\{\begin{array}{l}
A^{++} \in \Lambda\left(r_{s}, \check{G}\right) \Leftrightarrow g\left(r_{s} \mid \sigma_{s}\right)>\hat{G} \Leftrightarrow g\left(r_{s} \mid \sigma_{s}\right) \geq \hat{G} \wedge \neg g\left(r_{s} \mid \sigma_{s}\right) \leq \hat{G}, \\
A^{+} \in \Lambda\left(r_{s}, \check{G}\right) \Leftrightarrow g\left(r_{s} \mid \sigma_{s}\right) \geq \hat{G}, \\
A^{0} \in \Lambda\left(r_{s}, \check{G}\right) \Leftrightarrow g\left(r_{s} \mid \sigma_{s}\right)=\hat{G} \Leftrightarrow g\left(r_{s} \mid \sigma_{s}\right) \geq \hat{G} \wedge g\left(r_{s} \mid \sigma_{s}\right) \leq \hat{G}, \\
A^{-} \in \Lambda\left(r_{s}, \breve{G}\right) \Leftrightarrow g\left(r_{s} \mid \sigma_{s}\right) \leq \hat{G}, \\
A^{--} \in \Lambda\left(r_{s}, \check{G}\right) \Leftrightarrow g\left(r_{s} \mid \sigma_{s}\right)<\hat{G} \Leftrightarrow \neg g\left(r_{s} \mid \sigma_{s}\right) \geq \hat{G} \wedge g\left(r_{s} \mid \sigma_{s}\right) \leq \hat{G} .
\end{array}\right.
$$

In this way we assign the advice subset to the security $\check{S}$. The value $\Lambda\left(r_{s}, \check{G}\right)$ is called the investment recommendation. This recommendation may be used as valuable starting point for equity portfolio strategies. On the other hand, the weak point of the proposed choice function is omitting the fundamental analysis result and the behavioral factors impact. When analyzing the above choice function it is easy to see lack of strong boundary between the advice Buy and Accumulate and between the advice Reduce and Sell. Justification for distinguishing between these recommendations we can search on the basis of fundamental analysis and between the behavioral aspects of the investment process. 


\section{Investment recommendations dependent on fuzzy expected return}

Let us assume that considered security is represented by the pair $\left(\varrho_{s}(\cdot \mid \check{C}), \sigma_{s}^{2}\right)$, where $\varrho_{s}(\cdot \mid \check{C}) \in[0 ; 1]^{\mathbb{R}}$ is the expected return distribution. This distribution describes fuzzy expected return $\tilde{R}$ determined for current market price $\check{C}$. Fuzzy expected return may be dependent on fundamental and behavioural factors. ${ }^{2}$ Therefore, there shall be proposed advisor's counsels dependent on fuzzy expected return and return variance.

In the first step, we extend the profit index domain $\mathbb{R}^{2}$ to the set $[0 ; 1]^{\mathbb{R}} \times \mathbb{R}$. According to Zadeh's Extension Principle, the profit index value $\tilde{g}(\tilde{R} \mid \check{C})$ is described by its membership function $\gamma: \mathbb{R} \rightarrow[0 ; 1]$ determined as follows

$$
\gamma(x \mid \check{C})=\sup \left\{\varrho_{s}(r \mid \check{C}): x=g\left(r \mid \sigma_{s}\right)\right\}=\varrho_{s}\left(g^{-1}\left(r \mid \sigma_{s}\right) \mid \check{C}\right) .
$$

In this way we define profit fuzzy index $\tilde{g}(\cdot \mid \check{C}):[0 ; 1]^{\mathbb{R}} \times \mathbb{R} \rightarrow[0 ; 1]^{\mathbb{R}}$.

In the second step, we extend the advice choice function domain $\mathbb{R}^{2}$ to the set $[0 ; 1]^{\mathbb{R}} \times \mathbb{R}$. Under Zadeh's Extension Principle, the advice choice function value $\tilde{\Lambda}(\tilde{R}, \check{G})$ is described by its membership function $\lambda: \mathbb{A} \rightarrow$ $[0 ; 1]$ which due to the definition (12) is determined in following way

$$
\begin{gathered}
\lambda\left(A^{++}\right)=\sup \{\gamma(x \mid \check{C}): x \geq \check{G}\} \wedge(1-\sup \{\gamma(x \mid \check{C}): x \leq \check{G}\}), \\
\lambda\left(A^{+}\right)=\sup \{\gamma(x \mid \check{C}): x \geq \check{G}\}, \\
\lambda\left(A^{0}\right)=\sup \{\gamma(x \mid \check{C}): x \geq \check{G}\} \wedge \sup \{\gamma(x \mid \check{C}): x \leq \check{G}\}, \\
\lambda\left(A^{-}\right)=\sup \{\gamma(x \mid \check{C}): x \leq \check{G}\}, \\
\lambda\left(A^{--}\right)=\sup \{\gamma(x \mid \check{C}): x \leq \check{G}\} \wedge(1-\sup \{\gamma(x \mid \check{C}): x \geq \check{G}\}),
\end{gathered}
$$

In this way we define advice fuzzy choice function $\tilde{\Lambda}:[0 ; 1]^{\mathbb{R}} \times \mathbb{R} \rightarrow[0 ; 1]^{\mathbb{A}}$. The value $\tilde{\Lambda}(\tilde{R}, \check{G})$ is called imprecise investment recommendation. Moreover, the value $\lambda(A)$ may be interpreted as a degree in which the advice $A \in \mathbb{A}$ was chosen.

The expected return is replaced above by the fuzzy return which also takes into account fundamental behavioral aspects of decision making in finance. However, such an increase in cognitive value has a price. This price is imprecise formulation of investment recommendations. When we use imprecise image of security, we cannot precisely indicate the recommended action out of a set of advices. Each advice is thus recommended to some extent. The investor shifts some of the responsibility to advisers. For this reason, 
the investor restricts his or her choice of investment decisions to advice recommended to the greatest degree. In this way, the investor minimizes his or her individual responsibility for financial decision making. However, final investment decision will be made by the investor. Guided by own knowledge and intuition, the investor can choose the advice which is recommended in the lower degree. This problem was widely discussed in (Piasecki, 1990).

Let us note that we have here

$$
\begin{gathered}
\lambda\left(A^{++}\right)=\lambda\left(A^{+}\right) \wedge\left(1-\lambda\left(A^{-}\right)\right), \\
\lambda\left(A^{0}\right)=\lambda\left(A^{+}\right) \wedge \lambda\left(A^{-}\right), \\
\lambda\left(A^{--}\right)=\lambda\left(A^{-}\right) \wedge\left(1-\lambda\left(A^{+}\right)\right),
\end{gathered}
$$

This shows that the functions values $\lambda\left(A^{+}\right)$and $\lambda\left(A^{-}\right)$are sufficient to determine the imprecise investment recommendation. For this reason, when considering the specific method of advice choice, we will only determine these values. Let us note that the use of imprecisely estimated return will allow determining the differences between the advice Buy and Accumulate and between the advice Reduce and Sell.

In the next two sections some examples of methodologies for imprecise recommendations will be presented. There we limit our discussion to the case when the security return is described by means of a simple return rate given as follows

$$
\tilde{r}(\omega)=\frac{\tilde{V}_{t}(\omega)-\check{C}}{\check{C}}
$$

where all used symbols are defined in Section 2. All methodologies presented below are extensions of well know classical methodologies to the fuzzy case.

\section{Financial equilibrium criteria}

Each financial equilibrium model is given as the comparison of expected return from considered security and expected return of the market portfolio. We will consider fixed security $\check{S}$.

\subsection{Sharpe's Ratio}

We assume that there exists the risk free bond instrument represented by the pair $\left(r_{0}, 0\right)$ and the market portfolio represented by the pair $\left(r_{M}, \sigma_{M}^{2}\right)$. If the security $\dot{S}$ is represented by the pair $\left(r_{s}, \sigma_{s}^{2}\right)$, then Sharpe (1966) defines the profit index $g\left(\cdot \mid \sigma_{s}\right): \mathbb{R} \rightarrow \mathbb{R}$ and the limit value $\hat{G}$ as follows 
Krzysztof Piasecki

$$
\begin{gathered}
g\left(r \mid \sigma_{s}\right)=\frac{r-r_{0}}{\sigma_{s}}, \\
\hat{G}=\frac{r_{M}-r_{0}}{\sigma_{M}} .
\end{gathered}
$$

Sharpe's profit index estimates amount of the premium per overall risk unit. Sharpe's limit value is equal to the unit premium of the market portfolio risk.

If the security $\check{S}$ is represented by the pair $\left(\varrho_{s}(\cdot \mid \check{C}), \sigma_{s}^{2}\right)$ then profit fuzzy index is described by its membership function $\gamma: \mathbb{R} \rightarrow[0 ; 1]$ determined as follows

$$
\gamma(x \mid \check{C})=\sup \left\{\varrho_{s}(r \mid \check{C}): x=\frac{r-r_{0}}{\sigma_{s}}\right\}=\varrho_{s}\left(\sigma_{s} \cdot x+r_{0} \mid \check{C}\right) .
$$

In accordance with (15) and (17) we have here

$$
\begin{aligned}
\lambda\left(A^{+}\right) & =\sup \left\{\gamma(x \mid \check{C}): x \geq \frac{r_{M}-r_{0}}{\sigma_{M}}\right\}= \\
& =\sup \left\{\varrho_{s}(r \mid \check{C}): \frac{r-r_{0}}{\sigma_{s}} \geq \frac{r_{M}-r_{0}}{\sigma_{M}}\right\}, \\
\lambda\left(A^{-}\right) & =\sup \left\{\gamma(x \mid \check{C}): x \leq \frac{r_{M}-r_{0}}{\sigma_{M}}\right\}= \\
& =\sup \left\{\varrho_{s}(r \mid \check{C}): \frac{r-r_{0}}{\sigma_{s}} \leq \frac{r_{M}-r_{0}}{\sigma_{M}}\right\} .
\end{aligned}
$$

The values $\lambda\left(A^{++}\right), \lambda\left(A^{0}\right), \lambda\left(A^{--}\right)$are determined respectively by relationships (19), (20) and (21).

\subsection{Jensen's Alpha}

On the capital market we observe the risk-free return $r_{0}$ and the expected market return $r_{M}$. The security $\check{S}$ is represented by the pair $\left(r_{s}, \beta_{s}\right)$, where $\beta_{s}$ is the directional factor of the CAPM model assigned to this instrument. Jensen (1969) defines the profit index $g\left(\cdot \mid \sigma_{s}\right): \mathbb{R} \rightarrow \mathbb{R}$ and the limit value $\hat{G}$ as follows

$$
\begin{gathered}
g\left(r \mid \sigma_{s}\right)=r-\beta_{s} \cdot\left(r_{M}-r_{0}\right), \\
\hat{G}=r_{0} .
\end{gathered}
$$

Let us note that the above profit index $g\left(\cdot \mid \sigma_{s}\right)$ does not depend on the standard deviation $\sigma_{s}$. Jensen's profit index estimates amount of the premium 
of the market portfolio risk. Jensen's limit value is equal to the risk-free return.

If the security $\check{S}$ is represented by the pair $\left(\varrho_{s}(\cdot \mid \check{C}), \beta_{s}\right)$ then profit fuzzy index is described by its membership function $\gamma: \mathbb{R} \rightarrow[0 ; 1]$ determined as follows

$$
\begin{aligned}
\gamma(x \mid \check{C}) & =\sup \left\{\varrho_{s}(r \mid \check{C}): x=r-\beta_{s} \cdot\left(r_{M}-r_{0}\right)\right\}= \\
& =\varrho_{s}\left(x+\beta_{s} \cdot\left(r_{M}-r_{0}\right) \mid \check{C}\right) .
\end{aligned}
$$

In accordance with (15) and (17) we have here

$$
\begin{aligned}
\lambda\left(A^{+}\right) & =\sup \left\{\gamma(x \mid \check{C}): x \geq r_{0}\right\}= \\
& =\sup \left\{\varrho_{s}(r \mid \check{C}): r-\beta_{s} \cdot\left(r_{M}-r_{0}\right) \geq r_{0}\right\}, \\
\lambda\left(A^{-}\right) & =\sup \left\{\gamma(x \mid \check{C}): x \leq r_{0}\right\}= \\
& =\sup \left\{\varrho_{s}(r \mid \check{C}): r-\beta_{s} \cdot\left(r_{M}-r_{0}\right) \leq r_{0}\right\} .
\end{aligned}
$$

The values $\lambda\left(A^{++}\right), \lambda\left(A^{0}\right), \lambda\left(A^{--}\right)$are determined respectively by relationships (19), (20) and (21).

\subsection{Treynor's Ratio}

Let us assume that the assumptions are the same as the assumptions used in subsection 5.2. Additionally we assume that the security return is positively correlated with the market portfolio return. Treynor (1965) defines the profit index $g\left(\cdot \mid \sigma_{s}\right): \mathbb{R} \rightarrow \mathbb{R}$ and the limit value $\hat{G}$ as follows

$$
\begin{gathered}
g\left(r \mid \sigma_{s}\right)=\frac{r-r_{0}}{\beta_{s}}, \\
\hat{G}=r_{M}-r_{0} .
\end{gathered}
$$

Treynor's profit index estimates amount of the premium per market risk unit. Treynor's limit value is equal to the premium of the market risk.

If the security $\check{S}$ is represented by the pair $\left(\varrho_{s}(\cdot \mid \check{C}), \sigma_{s}^{2}\right)$ then profit fuzzy index is described by its membership function $\gamma: \mathbb{R} \rightarrow[0 ; 1]$ determined as follows

$$
\gamma(x \mid \check{C})=\sup \left\{\varrho_{s}(r \mid \check{C}): x=\frac{r-r_{0}}{\beta_{s}}\right\}=\varrho_{s}\left(\beta_{s} \cdot x+r_{0} \mid \check{C}\right) .
$$


In accordance with (15) and (17) we have here

$$
\begin{aligned}
\lambda\left(A^{+}\right) & =\sup \left\{\gamma(x \mid \check{C}): x \geq r_{M}-r_{0}\right\}= \\
& =\sup \left\{\varrho_{s}(r \mid \check{C}): \frac{r-r_{0}}{\beta_{s}} \geq r_{M}-r_{0}\right\}, \\
\lambda\left(A^{-}\right) & =\sup \left\{\gamma(x \mid \check{C}): x \leq r_{M}-r_{0}\right\}= \\
& =\sup \left\{\varrho_{s}(r \mid \check{C}): \frac{r-r_{0}}{\beta_{s}} \leq r_{M}-r_{0}\right\} .
\end{aligned}
$$

The values $\lambda\left(A^{++}\right), \lambda\left(A^{0}\right), \lambda\left(A^{--}\right)$are determined respectively by relationships (19), (20) and (21).

Investment recommendation made by means of the Treynor Ratio is identical with investment recommendation made by Jensen's Alpha.

\section{The Safety-First Criteria}

We will consider the simple return rate $\tilde{r}(\omega)$ on fixed security. For each assumed value $r \in \mathbb{R}$ of expected simple return rate the probability distribution of this return is given by the cumulative distribution function $F(\cdot \mid r): \mathbb{R} \rightarrow[0 ; 1]$ which is strictly increasing and continuous. Then the Safety Condition (Roy, 1952) is given in following way

$$
F(L \mid r)=\varepsilon,
$$

where:

- $L$ denotes minimum acceptable return rate;

$-\varepsilon$ is equal to probability realization of return below the minimum acceptable rate.

The realization of return below the minimum acceptable rate is identified with a loss. Therefore, the variable $\varepsilon$ denotes the loss probability.

Additionally, let us note that the function $G_{l}: \mathbb{R} \rightarrow[0 ; 1]$ defined for fixed $l \in R$ as follows

$$
G_{l}(r)=F(l \mid r)
$$

is strictly decreasing.

Let us take into account the security $\check{S}$ with expected return $r_{s}$. The distribution of this return is described by its cumulative distribution function $F_{s}\left(\cdot \mid r_{s}\right): \mathbb{R} \rightarrow[0 ; 1]$. It is obvious that the cumulative distribution function $F_{s}\left(\cdot \mid r_{s}\right)$ depends on the standard deviation $\sigma_{s}$. 


\subsection{Roy's Criterion}

Roy's Criterion (Roy, 1952) is that for fixed minimum acceptable return rate $L$ the investor minimizes the loss probability. Additionally in order to ensure financial security, the investor assumes the maximum level $\varepsilon^{*}$ of loss probability. Then the profit index $g\left(\cdot \mid \sigma_{s}\right): \mathbb{R} \rightarrow[-1 ; 0]$ and the limit value $\hat{G}$ are defined as follows

$$
\begin{gathered}
g\left(r \mid \sigma_{s}\right)=-G_{L}(r), \\
\hat{G}=-\varepsilon^{*} .
\end{gathered}
$$

If the security $\check{S}$ is represented by the pair $\left(\varrho_{s}(\cdot \mid \check{C}), \beta_{s}\right)$ then profit fuzzy index is described by its membership function $\gamma:[-1 ; 0] \rightarrow[0 ; 1]$ determined in following way

$$
\gamma(x \mid \check{C})=\sup \left\{\varrho_{s}(r \mid \check{C}): x=-G_{L}\right\}=\varrho_{s}\left(G_{L}^{-1}(-x) \mid \check{C}\right) .
$$

In accordance with (15) and (17) we have here

$$
\begin{aligned}
& \lambda\left(A^{+}\right)=\sup \left\{\gamma(x \mid \check{C}): x \geq-\varepsilon^{*}\right\}=\sup \left\{\varrho_{s}(r \mid \check{C}): F_{s}(L \mid r) \leq \varepsilon^{*}\right\}, \\
& \lambda\left(A^{-}\right)=\sup \left\{\gamma(x \mid \check{C}): x \leq-\varepsilon^{*}\right\}=\sup \left\{\varrho_{s}(r \mid \check{C}): F_{s}(L \mid r) \geq \varepsilon^{*}\right\} .
\end{aligned}
$$

The values $\lambda\left(A^{++}\right), \lambda\left(A^{0}\right), \lambda\left(A^{--}\right)$are determined respectively by relationships (19), (20) and (21).

\subsection{Kataoka's Criterion}

Kataoka's Criterion (Kataoka, 1963) is that, for fixed loss probability $\varepsilon$ the investor maximizes the minimum acceptable return rate. In addition, in order to ensure interest yield, the investor assumes the minimum level $L^{*}$ of return. Then the profit index $g\left(\cdot \mid \sigma_{s}\right):[0 ; 1] \rightarrow \mathbb{R}$ and the limit value $\hat{G}$ are defined in following way

$$
\begin{gathered}
g\left(r \mid \sigma_{s}\right)=F_{s}^{-1}(\varepsilon \mid r), \\
\hat{G}=L^{*} .
\end{gathered}
$$

If the security $\check{S}$ is represented by the pair $\left(\varrho_{s}(\cdot \mid \check{C}), \beta_{s}\right)$ then profit fuzzy index is described by its membership function $\gamma: \mathbb{R} \rightarrow[0 ; 1]$ determined as follows

$$
\gamma(x \mid \check{C})=\sup \left\{\varrho_{s}(r \mid \check{C}): x=F_{s}^{-1}(\varepsilon \mid r)\right\}=\varrho_{s}\left(G_{L}^{-1}(\varepsilon) \mid \check{C}\right) .
$$

In accordance with (15) and (17) we have here 
Krzysztof Piasecki

$$
\begin{aligned}
& \lambda\left(A^{+}\right)=\sup \left\{\gamma(x \mid \check{C}): x \geq L^{*}\right\}=\sup \left\{\varrho_{s}(r \mid \check{C}): F_{s}^{-1}(\varepsilon \mid r) \geq L^{*}\right\}, \\
& \lambda\left(A^{-}\right)=\sup \left\{\gamma(x \mid \check{C}): x \leq L^{*}\right\}=\sup \left\{\varrho_{s}(r \mid \check{C}): F_{s}^{-1}(\varepsilon \mid r) \leq L^{*}\right\} .
\end{aligned}
$$

The values $\lambda\left(A^{++}\right), \lambda\left(A^{0}\right), \lambda\left(A^{--}\right)$are determined respectively by relationships (19), (20) and (21).

\subsection{Telser's Criterion}

Based on the safety and profitability of the investment, the investor assumes a minimum level $L^{*}$ of acceptable return and the maximum level $\varepsilon^{*}$ of loss probability. If for the security $\breve{S}$ the probability that return is not acceptable is less than acceptable loss probability then $\check{S}$ is called safe-haven security.

Let the return on security $\check{S}$ be given as the fuzzy probabilistic set outlined in (8) by its membership function $\rho(\cdot \mid \check{C}): \mathbb{R} \times \Omega \rightarrow[0 ; 1]$. Then, for any minimum acceptable minimum return $L \in \mathbb{R}$ the loss is the fuzzy probabilistic set described by membership function $\eta(\cdot \mid L, \check{C}): \Omega \rightarrow[0 ; 1]$ determined as follows

$$
\begin{aligned}
\eta(\omega \mid L, \check{C}) & =\sup \{\rho(r, \omega \mid \check{C}): r<L\}= \\
& =\sup \left\{\omega\left(\tilde{V}_{t}(\omega) \cdot(1+r)^{-1} \mid \check{C}\right): r<L\right\} .
\end{aligned}
$$

In accordance with (3), for expected return $r_{s}$ on $\check{S}$ the probability distribution of future value is described by conditional cumulative distribution function $F_{V, S}\left(\cdot \mid r_{s}, \check{C}\right): \mathbb{R}^{+} \rightarrow[0 ; 1]$ determined in the following way

$$
F_{V, S}\left(x \mid r_{s}, \check{C}\right)=F_{s}\left(\frac{x-\check{C}}{\check{C}} \mid r_{s}\right) .
$$

In this case, due (4), (6) and (7), the loss probability $P_{s}(L)$ may be calculated as follows

$$
\begin{gathered}
P_{s}(L)=\int_{-\infty}^{+\infty} \sup \left\{\mu\left(x \cdot(1+r)^{-1} \mid \check{C}\right): r<L\right\} d F_{V, S}\left(x \mid r_{s}, \check{C}\right)= \\
=\int_{-\infty}^{+\infty} \sup \left\{\mu\left(\check{C} \cdot(1+t) \cdot(1+r)^{-1} \mid \check{C}\right): r<L\right\} d F_{s}\left(t \mid r_{s}\right)= \\
=\int_{-\infty}^{L} d F_{s}\left(t \mid r_{s}\right)+\int_{L}^{+\infty} \mu\left(\check{C} \cdot(1+t) \cdot(1+L)^{-1} \mid \check{C}\right) d F_{s}\left(t \mid r_{s}\right)= \\
=F_{s}\left(L \mid r_{s}\right)+\int_{L}^{T} \mu\left(\check{C} \cdot(1+t) \cdot(1+L)^{-1} \mid \check{C}\right) d F_{s}\left(t \mid r_{s}\right),
\end{gathered}
$$


where

$$
T=\frac{\check{C}_{\max }}{\check{C}} \cdot(1+L)-1 .
$$

The security $\check{S}$ is safe-haven one iff the following condition is fulfilled

$$
P_{s}\left(L^{*}\right) \leq \varepsilon^{*} .
$$

Telsers's Criterion (Telser, 1955) is that the investor maximizes the return on safe-haven securities. In addition, due to the profitability investment, the investor takes into account the equilibrium rate $r^{*}>L^{*}$. Then the profit index $g\left(\cdot \mid \sigma_{s}\right):[0 ; 1] \rightarrow \mathbb{R}$ and the limit value $\hat{G}$ are defined in the following way

$$
\begin{gathered}
g\left(r \mid \sigma_{s}\right)=r, \\
\hat{G}=r^{*} .
\end{gathered}
$$

If the security $\check{S}$ is represented by the pair $\left(\varrho_{s}(\cdot \mid \check{C}), \beta_{s}\right)$ then profit fuzzy index is described by its membership function $\gamma: \mathbb{R} \rightarrow[0 ; 1]$ determined as follows

$$
\gamma(x \mid \check{C})=\varrho_{s}(x \mid \check{C}) .
$$

In accordance with (15) and (17) we have here

$$
\begin{aligned}
& \lambda\left(A^{+}\right)=\sup \left\{\varrho_{s}(r \mid \check{C}): r \geq r^{*}\right\}, \\
& \lambda\left(A^{-}\right)=\sup \left\{\varrho_{s}(r \mid \check{C}): r \leq r^{*}\right\} .
\end{aligned}
$$

The values $\lambda\left(A^{++}\right), \lambda\left(A^{0}\right), \lambda\left(A^{--}\right)$are determined respectively by relationships (19), (20) and (21).

\section{Summary}

Imprecision is relevant to the investment process. It is shown above that imprecise estimate of the present value may be considered as a sufficient basis for determining the investment recommendation. The results so obtained may be applied in behavioural finance theory as a normative model of investment's decisions. These results may provide theoretical foundations for constructing an investment decision support system.

On the other hand imprecise estimate of the expected return could be a consequence of taking into account behavioral aspects of the investment 
process. Thus, we have shown here that the behavioral premises can influence investment recommendations in a controlled manner.

Applications of the above normative models cause several difficulties. The main difficulty is the high formal and computational complexity of the tasks involved in determining the membership function for the imprecise recommendations. The computational complexity of these models is the price we pay for the lack of detailed assumptions about the return rate. On the other hand, the low logical complexity is an important attribute of each formal model.

In this paper, the main cognitive result is to propose general methodology for imprecise investments recommendations. Moreover, the paper also offers original generalization of the financial equilibrium criteria and of the safety-first criteria to the fuzzy case.

\footnotetext{
N O T E S

1 Can find http://www.marketwatch.com/tools/guide.asp [access 01.09.2013].

2 For example (Piasecki, $2011 \mathrm{a}, \mathrm{b})$.
}

\section{R E F E R E N C E S}

Barberis, N., Shleifer, A., Vishny R., (1998). A model of investor sentiment. Journal of Financial Economics, 49, 307-343.

Bølviken, E., Benth, F.E., (2000). Quantification of risk in Norwegian stocks via the normal inverse Gaussian distribution, Proceedings of the AFIR 2000 Colloquium, Troms $\varnothing, 87-98$.

Buckley, J.J., (1987). Fuzzy mathematics of finance. Fuzzy Sets and Systems, 21, 257-273.

Buckley, J.J., (1992). Solving fuzzy equations in economics and finance. Fuzzy Sets and Systems, 48, 289-296.

Calzi, M.L. (1990). Towards a general setting for the fuzzy mathematics of finance. Fuzzy Sets and Systems, 35, 265-280.

Dacey, R., Zielonka P., (2005). A detailed prospect theory explanation of the disposition effect. Journal of Behavioral Finance, 2/4, 43-50.

Dubois, J., Prade, H., (1979). Fuzzy real algebra: some results. Fuzzy Sets and Systems, vol. 2.

Edwards, W., (1968). Conservatism in human information processing, In: Klienmutz B. (Ed) Formal representation of human judgment, New York: Wiley, $17-52$. 
Fang Yong, Lai Kin Keung, Wang Shouyang, (2008). Fuzzy portfolio optimization. Theory and methods, Lecture Notes in Economics and Mathematical Systems 609. Berlin: Springer.

Greenhut, J.G., Norman, G., Temponi, C., (1995). Towards a fuzzy theory of oligopolistic competition, IEEE Proceedings of ISUMA-NAFIPS 1995, 286291.

Gutierrez, I., (1989). Fuzzy numbers and Net Present Value. Scand. J. Mgmt, vol. 5 no $2,149-159$.

Hiroto, K., (1981). Concepts of probabilistic sets. Fuzzy Sets and Systems, 5, 31-46.

Huang, X., (2007). Two new models for portfolio selection with stochastic returns taking fuzzy information. European Journal of Operational Research, vol. 180 no 1, 396-405.

Jensen, M.C., (1969). Risk and pricing of capital assets, and the evaluation of investments portfolios. Journal of Business, vol. 42 no 2, 167-247.

Kaplan, S., Barish, N.N., (1967). Decision-Making Allowing Uncertainty of Future Investment Opportunities. Management Science, vol. 13 no 10, 569-577.

Kataoka, S., (1963). A stochastic programming model. Econometrica, vol. 31 no $1 / 2 ., 181-196$.

Knight, F. H. (1921). Risk, Uncertainty, and Profit. Boston, MA: Hart, Schaffner \& Marx; Houghton Mifflin Company.

Kuchta, D., (2000). Fuzzy capital budgeting, Fuzzy Sets and Systems, 111, 367-385.

Kwakernaak, H.K., (1978). Fuzzy random variables I, Information Sciences, 15, $1-29$.

Kwakernaak, H.K., (1979). Fuzzy random variables II, Information Sciences, 17, 253-278.

Lesage, C., (2001). Discounted cash-flows analysis. An interactive fuzzy arithmetic approach. European Journal of Economic and Social Systems vol. 15 no 2, 49-68.

Mises, L. von, (1962), The Ultimate Foundation of Economic Science An Essay on Method, Princeton: D. Van Nostrand Company, Inc.

Piasecki, K., (1990). Decyzje $i$ wiarygodne prognozy. Zeszyty Naukowe Akademii Ekonomicznej S. II z. 106, Poznań.

Piasecki, K., (2011a). Behavioural Present Value, Behavioral \& Experimental Finance eJournal" 2011/4, [online]: http://ssrn.com/abstract=1729351.

Piasecki, K., (2011b). Rozmyte zbiory probabilistyczne jako narzędzie finansów behawioralnych, Poznań: Wydawnictwo Uniwersytetu Ekonomicznego.

Piasecki, K. (2011c). Effectiveness of securities with fuzzy probabilistic return. Operations Research and Decisions, 2/2011, 65-78.

Piasecki, K. (2012). Basis of financial arithmetic from the viewpoint of the utility theory, Operations Research and Decisions, 03/2012, 37-53. 
Piasecki, K. (2013). Imprecise return rates on the Warsaw Stock Exchange, Proceedings of International Colloquium on Current Economics and Social Topics CEST'2013, (in print).

Piasecki, K., Tomasik, E. (2013). Rozkłady stop zwrotu z instrumentów polskiego rynku kapitałowego, edu-Libri, Kraków-Warszawa.

Roy, A.D., (1952). Safety-first and the holding of assets, Econometrics, 20, 431-449.

Sharpe, W.F., (1966). Mutual fund performance. Journal of Business, 19, 119-138.

Sheen, J.N., (2005). Fuzzy financial profitability analyses of demand side management alternatives from participant perspective. Information Sciences, 169, 329-364.

Telser, L.G. (1955). Safety first and hedging. The Review of Economic Studies, vol. 23 no $2,1-16$.

Treynor, J.L., (1965). How to rate management of investment fund. Harvard Business Review, 43, 63-75.

Tsao, C.-T., (2005). Assessing the probabilistic fuzzy Net Present Value for a capital, Investment choice using fuzzy arithmetic, J. of Chin. Ins. of Industrial Engineers, vol. 22 no 2, 106-118.

Ward, T.L., (1985). Discounted fuzzy cash flow analysis, 1985 Fall Industrial Engineering Conference Proceedings, 476-481. 